\title{
Transplantation of Encapsulated Dopamine-Secreting Cells as a Treatment for Parkinson's Disease
}

\author{
D.F. Emerich, T.R. Flanagan, B.R. Frydel, F.T. Gentile, M.A. Palmatier and S.R. Winn \\ CytoTherapeutics Inc., 2 Richmond Square, Providence, RI 02906, USA
}

Cellular transplantation is rapidly becoming recognized as a possible therapeutic approach in human neurodegenerative disorders such as Parkinson's disease (PD). The transition from basic research to broad scale clinical application requires the clear demonstration of the safety of this technology. A milestone in this transition involves the identification of suitable cells for transplantation. The use of dopaminergic tissue derived from fetal or adrenal sources has been explored in transplantation as a possible longterm treatment for PD. In this approach, several issues remain unresolved. First there has been an inability to demonstrate long-term survival of transplanted tissue with consistent functional recovery in the clinical trials conducted to date. Furthermore, the availability of donor tissue, the amount of tissue needed to produce functional recovery, the safety of the cell source, and the ethical perception of using human fetal tissue remain significant obstacles to the use of these tissues.

The use of non-human donor cells provides distinct advantages over human fetal cells as a source of therapeutically transplanted cells. We are developing a cellular transplant therapy for PD based on the use of polymer encapsulation to immuno-isolate animal cells. The cells we currently employ, PC12 cells, are an established tissue culture line initially isolated from a rat adrenomedullary chromaffin cell tumor. Transplantation of encapsulated PC12 cells provides specific safety advantages: the cells are rapidly destroyed by the immune system if they escape from immuno-isolation, the cells are free from microbial and viral infections, and the cells are grown under controlled conditions so that each lot of cells will be from uniform, defined growth stages. The key element to the successful appli- cation of this technology lies in the immunoisolation of the cells. Cells are immuno-isolated by surrounding them with a permselective polymer membrane that allows nutrients to enter the capsule so that the cells will survive and function. Additionally, the membrane is fabricated such that the molecular weight cutoff allows dopamine and other low molecular weight molecules to leave the capsule.

Cellular encapsulation within polymeric hollow fibers can be achieved in several ways: cells can be hand-loaded into pre-formed hollow fibers or cells can be co-extruded with polymer so that a continuous polymeric fiber forms around the living cells. Both of these processes require additional steps in which fibers are cut to capsule size specifications and the ends of these capsules are sealed. We have additionally developed a semi-automated, computer-controlled version of the co-extrusion approach suitable for rapidly manufacturing integrally sealed capsules. This approach is rapid and its reproducibility and safety is assured through hands-off process validation.

Immuno-isolated capsules prepared from polyacrylonitrile-vinyl chloride copolymer (PAN/PVC) using sterile fabrication processes are biocompatible following striatal implantation. Analysis of host tissue at the implant site using light and electron microscopy has shown that polymer capsules implanted into the rodent or primate striatum produce a mild gliotic reaction that rapidly diminishes over time. Inclusion of PC12 cells within the capsule does not affect the biocompatibility, suggesting that the encapsulated cells do not produce, or that the capsule retains, physiologically active levels of inflammatory or immune mediators. 
Cellular encapsulation offers additional safety features not available in approaches using unencapsulated cells. Encapsulation enables the use of qualified cell lines. Thus, cells can be used which will grow under quarantine culture conditions allowing sufficient time to meet FDA guidelines for safety. Encapsulation enables dosage control: cells can be either loaded to prescribed densities (non-proliferating cell types) or seeded to grow-up to fill a finite internal volume (proliferating cell types). Encapsulation insures that transplanted cells are also retrievable and can be rapidly and safely removed in their entirety as therapeutically required. Finally, appropriately selected cells (i.e. animal cells) which escape encapsulation will not survive unencapsulated.

The ultimate measure of the therapeutic utility of encapsulated cells is in efficacy testing. Encapsulated PC12 cells ameliorate apomorphine-induced rotation behavior and sensori- motor deficits in 6-OHDA lesioned rats following implantation into the denervated striatum. The deficits in motor coordination and balance which occur in aged rats are also partially reversed by the bilateral placement of encapsulated PC12 cells into the striatum. Aebischer and colleagues reported that encapsulated PC12 cells produced behavioral recovery in a unilateral MPTP-treated primate. After intrastriatal implantation of PC12 cells, the monkey's impaired performance in a motor dexterity model improved significantly and the extent of MPTP-induced tremor was reduced.

Pending the successful completion of additional preclinical efficacy and safety studies in non-human primates we believe that the implantation of encapsulated dopamine-secreting cells into PD patients may provide an effective means of alleviating the symptoms of the disease. Furthermore, the technique of encapsulation has applications in diseases other than PD that are characterized by secretory cell dysfunction. 

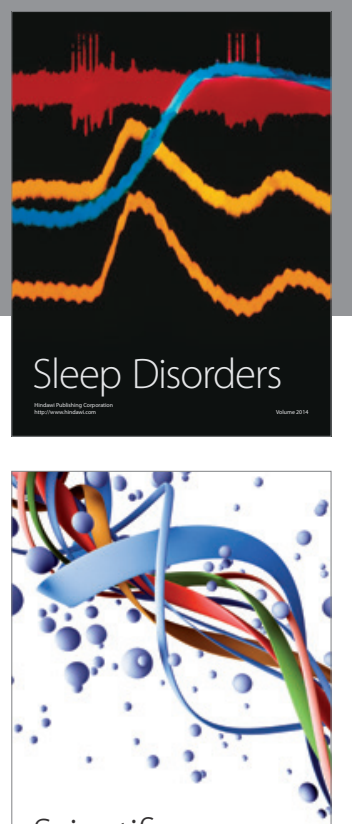

Scientifica
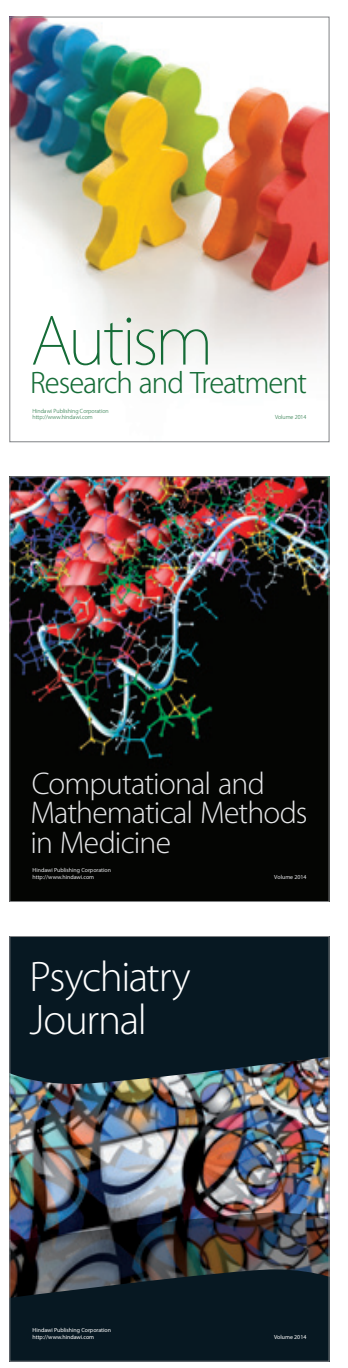
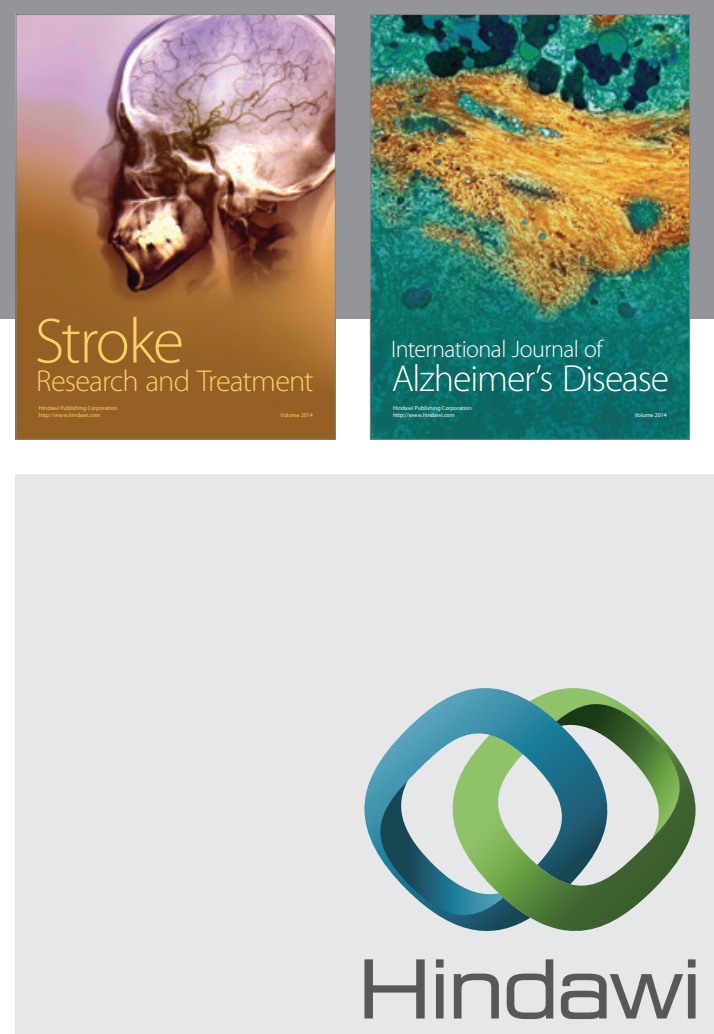

Submit your manuscripts at

http://www.hindawi.com
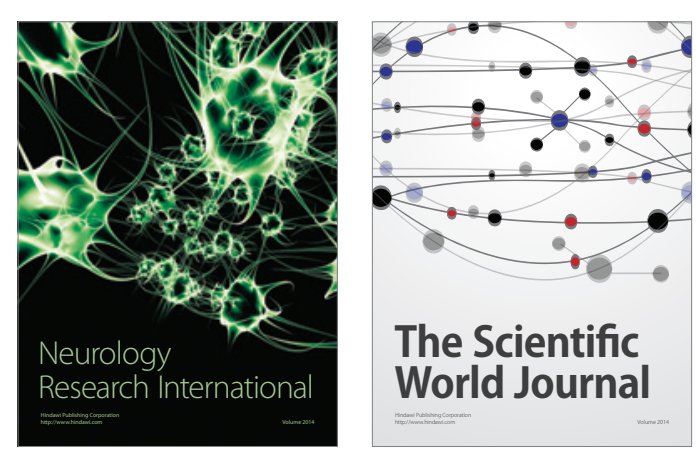

The Scientific World Journal

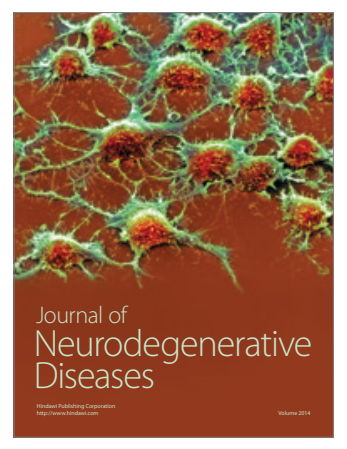

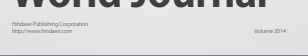

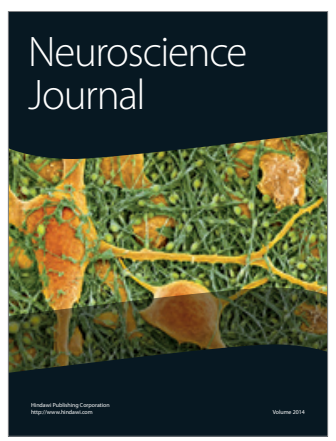

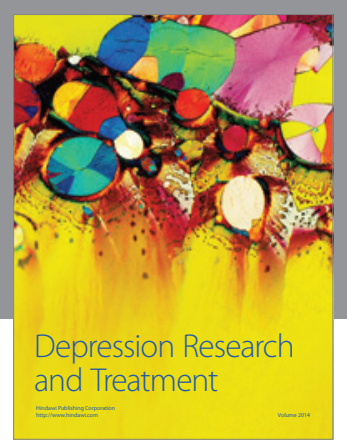
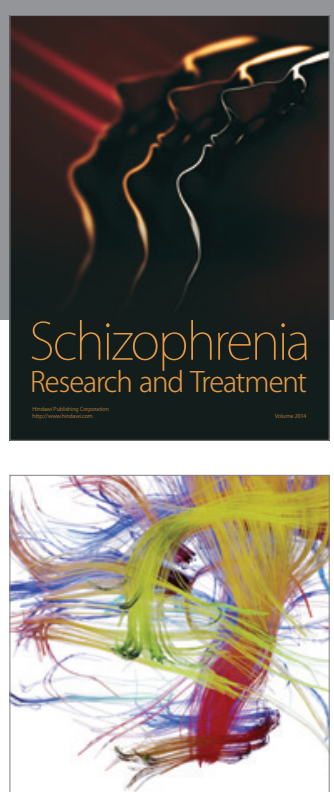

Brain Science

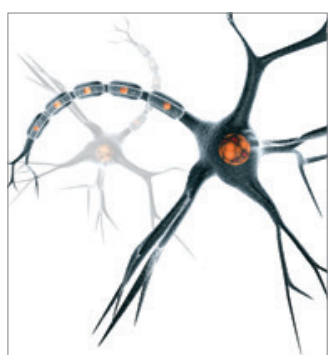

Neural Plasticity
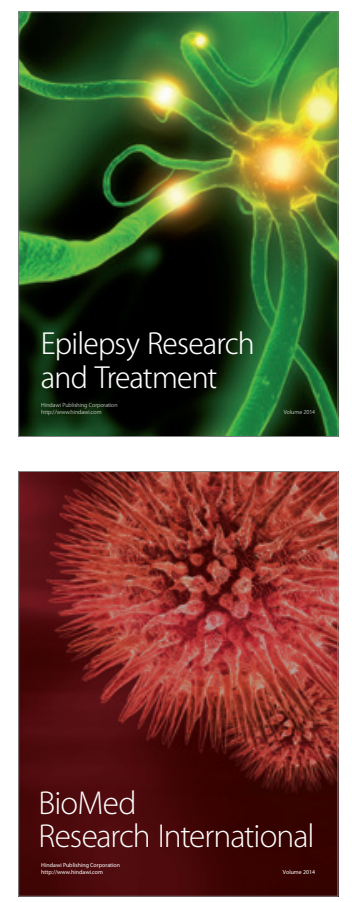

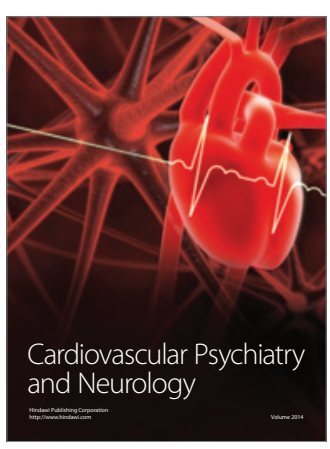

Parkinson's

Disease
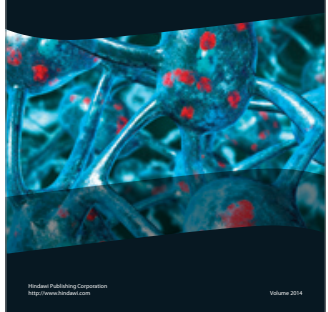